rheumatoid arthritis (RA) [2]. Therefore, neutralizing IL-17 might be a therapeutic option during the asymptomatic autoimmune prodromal phase in autoimmune diseases like RA, where TH17 cytokines orchestrate the emergence of a pro-inflammatory autoantibody response and the transition into active RA.

REFERENCES:

[1] McInnes IB, G. Schett, The pathogenesis of rheumatoid arthritis. N Engl J Med 2011; 365: 2205-19.

[2] Pfeifle $R$ et al, Regulation of autoantibody activity by the IL-23-Th17 axis determines the onset of autoimmune disease. Nat Immunol. 2017, Jan;18(1):104-113.

Disclosure of Interests: Rene Pfeifle Grant/research support from: Novartis AG., Julia Kittler: None declared, Manfred Wuhrer: None declared, Georg Schett: None declared, Gerhard Krönke Grant/research support from: Novartis AG DOI: 10.1136/annrheumdis-2021-eular.1087

\section{AB0017 IMMUNE CHARACTERISTICS OF PERIPHERAL BLOOD IN SECONDARY SJOGREN'S SYNDROME PATIENTS WITH RHEUMATOID ARTHRITIS}

R. Su ${ }^{1}$, Y. Y. Wang ${ }^{1}$, C. Wang ${ }^{1}{ }^{1}$ The Second Hospital of Shanxi Medical University, Rheumatology, Taiyuan, China

Background: Secondary Sjogren's Syndrome (sSS) is diagnosed when symptoms of SS coexist with other systemic connective tissue disease, often secondary to rheumatoid arthritis(RA).The occurrence of SS secondary with RA will worsen the course of disease and increase the high incidence and mortality of RA. At present, the immune characteristics of peripheral blood of sSS with RA are not clear. Objectives: To observe the difference of immune Immune characteristics in peripheral blood between sSS secondary to RA, primary Sjogren's syndrome(pSS) and RA patients

Methods: 20 sSS with RA patients, 20 pSS paients and 20 RA pateints hospitalized in ShanXi medical university the second Hospital were enrolled. The percentage and absolute numbers of lymphocyte phenotypes and CD4+ T subsets in peripheral blood were examined by flow cytometry.

Results: As for the percentage and absolute number of total $T, B, N K$ $C D 4+T, C D 8+T$ and the ratio of $C D 4+T$ to $C D 8+T$ cells, there was no significant difference between the sSS with RA, RA, and SS group. There was also no statistical difference in the percentage of CD4+T subsets(Th1,Th2,Th17 and Treg) between the three groups. But the ratio of Th17 and Treg in sSS with RA group was increased than pSS group.About comparison of absolute number of CD4+T subsets, there was no statistical difference among the three groups except that the Th1 cells in RA group was significantly higher than SS group.

Conclusion: Elevated Th17/Treg may be an immunological feature that differentiates SSS with RA patients from pSS patients. In addition, in general, peripheral blood of patients with RA and SS have similar immune characteristics. REFERENCES

[1] Wei W,Ahmad S S, Chi S. From Molecular Mechanism to the Etiology of Sjogren Syndrome.Curr Pharm Des. 2018;24(35):4177-4185

[2] Hajiabbasi A, Masooleh I S,Alizadeh Y, Secondary Sjogren's Syndrome in 83 Patients With Rheumatoid Arthritis.Acta Med Iran.2016;54(7):448-53.
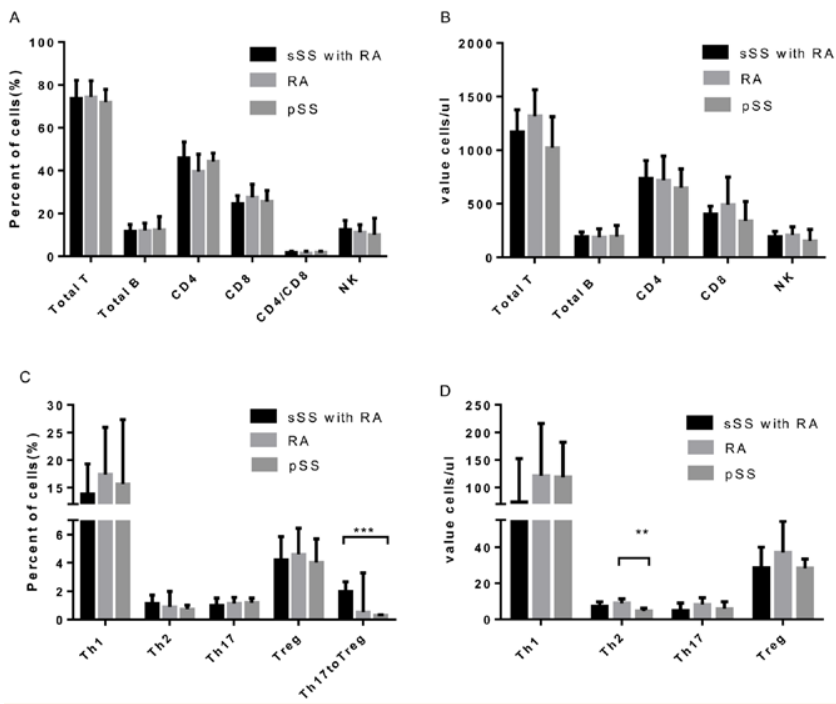

Figure 1. The comparsion about the lymphocyte phenotypes and CD4+ T subsets in peripheral blood of sSS with $R A(n=20), p S S(n=20)$ and RA patients $(n=20) .\left({ }^{*} p<0.05,{ }^{* *} p<0.001,{ }^{*} p<0.0001\right)$

Disclosure of Interests: None declared

DOI: 10.1136/annrheumdis-2021-eular.1158

\section{$\mathrm{AB} 0018$}

ACCUMULATION OF FUNCTIONALLY MATURE CD1C+ DENDRITIC CELLS CONTRIBUTES TO SYNOVIAL INFLAMMATION IN INFLAMMATORY ARTHRITIS

M. Canavan ${ }^{1,2}$, V. Marzaioli ${ }^{1,2}$, V. Bhargava ${ }^{3}$, S. Nagpal ${ }^{3}$, P. Gallagher ${ }^{4}$ C. Hurson ${ }^{4}$, R. Mullan ${ }^{5}$, D. Veale ${ }^{2}$, U. Fearon ${ }^{1,2} .{ }^{1}$ Trinity College Dublin, Molecular Rheumatology, Dublin, Ireland; ${ }^{2}$ St Vincent's University Hospital, Centre for Arthritis and Rheumatic Diseases, Dublin, Ireland; ${ }^{3} J a n s s e n$ Research and Development, Immunology and Discovery Sciences, Pennsylvania, United States of America; ${ }^{4}$ St Vincent's University Hospital, Department of Rheumatology, Dublin, Ireland; ${ }^{5}$ Adelaide and Meath Hospital, Department of Rheumatology, Dublin, Ireland

Background: Myeloid Dendritic Cells (DC) are potent antigen presenting cells that can be subdivided into $\mathrm{CD} 141$ and $\mathrm{CD} 1 \mathrm{c}^{+} \mathrm{DC}$. We have previously reported an unacknowledged role for $\mathrm{CD} 141^{+} \mathrm{DC}$ in the IA synovium. However, the identification and function of $C D 1 c^{+} D C$ in the IA synovium has yet to be fully elucidated. Objectives: To investigate if $C D 1 c^{+} D C$ reside in the IA synovium and ascertain if they represent a unique population, distinct from peripheral $C D 1 c^{+} D C$ and if they contribute to synovial inflammation.

Methods: Synovial tissue (ST) biopsies and synovial fluid mononuclear cells (SFMC) were obtained via arthroscopy and healthy control (HC) ST was obtained during ACL surgery. Synovial tissue single cells suspensions were generated following enzymatic and mechanical digestion. Single cell analysis of synovial tissue cell suspensions, along with PBMC and SFMC was performed by multicolour flow cytometry. $C D 1 C^{+} D C$ were sorted from IA synovial fluid and peripheral blood and bulk RNA sequencing was performed. $C D 1 c^{+} D C$ functionality and maturation was assessed using OVA DQ phagocytosis assays, multiplex ELISA and DC: T cell cocultures.

Results: Within the circulation the frequency of $C D 1 c^{+} D C$ are significantly decreased in IA peripheral blood compared to $\mathrm{HC}(\mathrm{p}<0.01)$ in addition to expressing significantly higher levels of the maturation markers CD80 $(p<0.01)$ and $C D 40(p=0.08)$. IA peripheral blood $D C$ also express significantly higher levels of CXCR3 $(p<0.01)$ and CCR7 $(p<0.05)$ compared to HC - suggestive of $\mathrm{DC}$ migration from the periphery to the synovium. Following RNA-seq analysis, IPA and differentially expressed gene (DEG) analysis revealed an enrichment in genes involved in DC maturation, TLR signalling and chemokine signalling in IA peripheral blood compared to $\mathrm{HC}$. In support of the hypothesis that DC migrate and accumulate in the IA synovium, CD1 $\mathrm{C}^{+} \mathrm{DC}$ were identified in IA ST and were significantly enriched compared to IA peripheral blood $(p<0.01)$. IA ST CD1 $c^{+} D C$ express significantly higher levels of the activation marker CD80 compared to IA peripheral blood $(p<0.05)$ or HC ST $(p<0.05)$. Upon examination of IA synovial fluid, we report similar findings to $S T$, whereby $C D 1 c^{+} D C$ are enriched in synovial fluid compared to PB $(p<0.001)$. Moreover, RNA sequencing and PCA analysis of synovial versus blood $C D 1 C^{+} D C$ revealed distinct transcriptional variation between both sites. Functionally, synovial $C D 1 c^{+} D C$ express higher levels of the maturation markers CD80, CD83, CD40, PD-L1 and BTLA (all $p<0.05$ ) and have distinct coexpression of these maturation markers which is unique to the synovium. Synovial $C D 1 c^{+} D C$ are less phagocytic compared to peripheral blood DC, have decreased production of MMP1 and MMP9 and importantly are still capable of additional activation in-vitro. Finally, synovial CD1c $c^{+} D C$ induce the proinflammatory cytokines TNF $\alpha$, GMCSF, IL-17a and IFN $\gamma$ from CD4 ${ }^{+}$T-cells in allogeneic DC: $T$ cells cocultures.

Conclusion: Mature circulatory $C D 1 c^{+} D C$ migrate and accumulate in the IA synovium. Synovial DC are present in the IA synovium in a mature state, have distinct tissue specific characteristics and can induce proinflammatory $C D 4^{+} T$ cell responses.

Acknowledgements: We would like to thank all the patients who contributed to this study

Disclosure of Interests: Mary Canavan: None declared, Viviana Marzaioli: None declared, Vipul Bhargava Employee of: Janssen Research and Development, Sunil Nagpal Employee of: Janssen Research and Development, Phil Gallagher: None declared, Conor Hurson: None declared, Ronan Mullan: None declared, Douglas Veale Speakers bureau: Abbvie, Janssen, Novartis, Pfizer, MSD, UCB, Consultant of: Abbvie, Janssen, Novartis, Pfizer, MSD, UCB, Grant/ research support from: Pfizer, Janssen, AbbVie, UCB, Ursula Fearon Speakers bureau: Abbvie, Grant/research support from: Pfizer, Janssen, Abbvie, UCB DOI: 10.1136/annrheumdis-2021-eular.1584

\section{$\mathrm{AB} 0019$ \\ TREATMENT OF AUTOIMMUNE AND INFLAMMATORY SKIN DISEASES USING SKIN-TARGETING BIFUNCTIONAL ANTIBODIES: A LOCALIZED IMMUNOMODULATION APPROACH}

S. Borthakur ${ }^{1}$, P. Mande ${ }^{2}$, D. Rios ${ }^{2}$, P. Halvey², A. Boisvert ${ }^{1}$, M. Rowe ${ }^{2}$ A. Agrawal ${ }^{1}$, M. Borah ${ }^{2}$, M. Cianci ${ }^{2}$, J. L. Viney ${ }^{2}$, K. Kis-Toth ${ }^{2}$, K. L. Otipoby², I. Mascanfroni ${ }^{2}$, N. Higginson-Scott ${ }^{1} .{ }^{1}$ Pandion Therapeutics Inc., Biotherapeutics, Watertown, United States of America; ${ }^{2}$ Pandion Therapeutics Inc., Immunology, Watertown, United States of America 